\title{
Monotone iterative techniques to SIRS epidemic models with nonlinear incidence rates and distributed delays
}

\author{
Yoshiaki Muroya $^{\mathrm{a}, *}$, Yoichi Enatsu ${ }^{\mathrm{b}}$, Yukihiko Nakata ${ }^{\mathrm{b}, \mathrm{c}}$ \\ ${ }^{a}$ Department of Mathematics, Waseda University 3-4-1 Ohkubo, Shinjuku-ku, Tokyo \\ 169-8555, Japan \\ ${ }^{b}$ Department of Pure and Applied Mathematics, Waseda University, 3-4-1 Ohkubo, \\ Shinjuku-ku, Tokyo, 169-8555, Japan \\ ${ }^{c} B C A M$ - Basque Center for Applied Mathematics Bizkaia Technology Park, Building 500, \\ E-48160, Derio, Basque Country, Spain
}

\begin{abstract}
In this paper, for SIRS epidemic models with a class of nonlinear incidence rates and distributed delays of the forms $\beta S(t) \int_{0}^{h} k(\tau) G(I(t-\tau)) d \tau$, we establish the global asymptotic stability of the disease-free equilibrium $E_{0}$ for $R_{0}<1$, and applying new monotone techniques, we obtain sufficient conditions which ensure the global asymptotic stability of the endemic equilibrium of system. The obtained results improve that in Xu and Ma [Stability of a delayed SIRS epidemic model with a nonlinear incidence rate, Chaos, Solitons and Fractals. 41 (2009) 2319-2325], and are very useful for a large class of SIRS models.
\end{abstract}

Keywords: SIRS epidemic model; nonlinear incidence; global asymptotic stability; distributed delays

\section{Introduction}

A fundamental problem in epidemic models is to study the global dynamics of disease transmissions, that is, to study the long term behavior of spread of the diseases. Various mathematical models have been proposed in the study of population dynamics and epidemiology. The incidence of a disease is the number of new cases per unit time and has played an important role in the literacy of mathematical modeling. Many authors have studied the dynamical behavior of several epidemic models (see [1-19] and references therein).

Much attention has been paid to the analysis of the stability of the diseasefree equilibrium and the endemic equilibrium of the epidemic models. Mena-

\footnotetext{
${ }^{*}$ Corresponding author.

Email addresses: ymuroya@waseda.jp (Yoshiaki Muroya), yo1.gc-rw.docomo@akane.waseda.jp (Yoichi Enatsu), yunayuna.na@gmail.com, nakata@bcamath.org (Yukihiko Nakata)

${ }^{a}$ Research is partially supported by Scientific Research (c), No.21540230 of Japan Society for the Promotion of Science.
}

Preprint submitted to Elsevier

December 10, 2010 
Lorca and Hethcote [11] considered an SIR epidemic model with bilinear incidence rate and no delays which takes the form $\beta S I$. Threshold was also found in Mena-Lorca and Hethcote [11] to determine whether the disease dies out or approaches to an endemic equilibrium. Later, various kinds of SIRS epidemic models and a significant body of work have been carried out (see, for example, $[6,8,13,17]$ and references therein).

Incidence rate plays a crucial role in the modeling of epidemic dynamics. Many authors have suggested that transmission of the infection shall have a nonlinear incidence rate. The bilinear incidence rate $\beta S I$ and the standard incidence rate $\beta S I / N$ are frequently used in the literacy of mathematical modeling. On the other hand, Capasso and Serio [3] have given an assumption that the incidence rate takes the nonlinear form $\frac{\beta S I}{1+\alpha I}$, which has been interpreted as saturated incidence rate. This incidence rate seems more reasonable than the bilinear incidence rate $\beta S I$ in the meaning that it includes the behavioral change and crowding effect of the infective individuals and prevents the unboundedness of the contact rate. For the following SIRS epidemic model with a nonlinear incidence rate and time delay;

$$
\left\{\begin{aligned}
\frac{d S(t)}{d t} & =B-\mu S(t)-\beta S(t) \frac{I(t-\tau)}{1+\alpha I^{p}(t-\tau)}+\delta R(t), \\
\frac{d I(t)}{d t} & =\beta S(t) \frac{I(t-\tau)}{1+\alpha I^{p}(t-\tau)}-(\mu+\gamma) I(t), \\
\frac{d R(t)}{d t} & =\gamma I(t)-(\mu+\delta) R(t),
\end{aligned}\right.
$$

$\mathrm{Xu}$ and Ma [17] for $p=1$ (saturation effect), Xiao and Ruan [16] for $p=2$ and Yang and Xiao [18] for $p>1$ (psychological effect), derived sufficient conditions for the global asymptotic stability of the endemic equilibrium.

In this paper, motivated by the above results and applying monotone techniques, we establish sufficient conditions which ensure the global asymptotic stability of endemic equilibrium of the following SIRS epidemic model (1.2) with a class of nonlinear incidence rates and distributed delays. The obtained results for (1.1) improve those in Xu and Ma [17] for $p=1$ and Yang and Xiao [18] for $p>1$.

$$
\left\{\begin{array}{l}
\frac{d S(t)}{d t}=B-\mu S(t)-\beta S(t) \int_{0}^{h} k(\tau) G(I(t-\tau)) d \tau+\delta R(t), \\
\frac{d I(t)}{d t}=\beta S(t) \int_{0}^{h} k(\tau) G(I(t-\tau)) d \tau-(\mu+\gamma) I(t) \\
\frac{d R(t)}{d t}=\gamma I(t)-(\mu+\delta) R(t)
\end{array}\right.
$$

where $S(t), I(t)$ and $R(t)$ denote the numbers of susceptible, infective and recovered individuals at time $t$, respectively. $B$ is the recruitment rate of the population, and $\mu$ is the natural death rate of the susceptible, infective and recovered individuals, $\beta$ is the transmission rate, $\gamma$ is the natural recovery rate 
of the infective individuals, $\delta$ is the rate at which recovered individuals lose immunity and return to the susceptible class. $\tau$ is the time taken to become infectious. $G(I(t-\tau))$ is a nonlinear incidence rate with a delay $\tau$ and $k(\tau)$ denotes the nonnegative incubation period distribution (see also Takeuchi et al. $[14])$. We assume that $G(I)$ is continuous on $(0,+\infty)$ and $k(t)$ is continuous on $[0, h]$ and

$$
\int_{0}^{h} k(\tau) d \tau=1
$$

The initial condition for system (1.2) takes the form

$$
\left\{\begin{array}{l}
S(\theta)=\phi_{1}(\theta), \quad I(\theta)=\phi_{2}(\theta), \quad R(\theta)=\phi_{3}(\theta) \\
\phi_{i}(\theta) \geq 0, \quad \theta \in[-h, 0], \quad \phi_{i}(0)>0, i=1,2,3 \\
\left(\phi_{1}(\theta), \phi_{2}(\theta), \phi_{3}(\theta)\right) \in C\left([-h, 0], \mathbb{R}_{+}^{3}\right)
\end{array}\right.
$$

where $\mathbb{R}_{+}^{3}=\left\{\left(x_{1}, x_{2}, x_{3}\right): x_{i} \geq 0, i=1,2,3\right\}$. We further assume that

$$
\left\{\begin{array}{l}
I / G(I) \text { is strictly monotone increasing on }(0,+\infty), \\
\text { and } \lim _{I \rightarrow+0}(I / G(I))=1 .
\end{array}\right.
$$

Notice that system (1.2) is a generalized form of systems (1.1), and from the condition (1.4), one can see that $G(I) \leq I$ for $I>0$ and $G(0)=0$. We note that $G(I)=\frac{I}{1+\alpha I}, \alpha>0$ satisfies the above condition (1.4) and the reproduction number for system (1.2) becomes

$$
R_{0}=\frac{\beta B}{\mu(\mu+\gamma)}
$$

It is well known by the fundamental theory of differential equations that system (1.2) has a unique solution $(S(t), I(t), R(t))$ satisfying initial condition (1.3). One can see immediately that system (1.2) always has a disease-free equilibrium $E_{0}=(B / \mu, 0,0)$. Apart from the above equilibrium, if $R_{0}>1$, then system (1.2) allows a unique endemic equilibrium $E_{*}=\left(S^{*}, I^{*}, R^{*}\right)$ satisfying the following.

$$
\left\{\begin{array}{l}
B-\mu S^{*}-\beta S^{*} G\left(I^{*}\right)+\delta R^{*}=0 \\
\beta S^{*} G\left(I^{*}\right)-(\mu+\gamma) I^{*}=0 \\
\gamma I^{*}-(\mu+\delta) R^{*}=0
\end{array}\right.
$$

Under the condition (1.4), let us define the following strictly monotone increasing function of $I>0$ such that

$$
h(I)= \begin{cases}\frac{I}{G(I)}, & \text { if } I>0, \\ 1, & \text { if } I=0,\end{cases}
$$

and consider its inverse function $h^{-1}(I)$ of $I \geq 0$. By (1.4), it holds that $h(0)=1$, and hence, $h^{-1}(1)=0$. Moreover, for any $0 \leq \underline{I} \leq \bar{I}$, put

$\bar{G}(\underline{I}, \bar{I})=\max _{\underline{I} \leq I \leq \bar{I}} G(I)= \begin{cases}G(\bar{I}), & \text { if } G(I) \text { is monotone increasing on }[\underline{I}, \bar{I}], \\ G(\hat{\bar{I}}), & \text { if there exists a maximal point } \hat{\bar{I}} \text { on }[\underline{I}, \bar{I}], \\ G(\underline{I}), & \text { if } G(I) \text { is monotone decreasing on }[\underline{I}, \bar{I}],\end{cases}$ 
and

$$
\underline{G}(\underline{I}, \bar{I})=\min _{\underline{I} \leq I \leq \bar{I}} G(I)= \begin{cases}G(\underline{\underline{I}}), & \text { if } G(I) \text { is monotone increasing on }[\underline{I}, \bar{I}], \\ G(\underline{\underline{I}}), & \text { if there exists a minimal point } \hat{I} \text { on }[\underline{I}, \bar{I}], \\ G(\bar{I}), & \text { if } G(I) \text { is monotone decreasing on }[\underline{I}, \bar{I}],\end{cases}
$$

and

$$
\bar{h}(\underline{I}, \bar{I})=\frac{\bar{I}}{\bar{G}(\underline{I}, \bar{I})}, \quad \text { and } \quad \underline{h}(\underline{I}, \bar{I})=\frac{\underline{I}}{\underline{G}(\underline{I}, \bar{I})} .
$$

We first obtain the similar result for $R_{0}<1$ in $\mathrm{Xu}$ and Ma [17, Proof of Theorem 3.2].

Theorem 1.1 If $R_{0}<1$, then the disease-free equilibrium $E_{0}$ of system (1.2) is globally asymptotically stable in the interior of $\mathbb{R}_{+}^{3}$.

We obtain the following main theorem (cf. Xu and Ma [17, Proof of Theorem $3.1])$.

Theorem 1.2 Let $R_{0}>1$. Then the positive equilibrium $E_{*}=\left(S^{*}, I^{*}, R^{*}\right)$ of system (1.2) exists. Assume that there exist two nonnegative constants $\underline{I}<\bar{I}$ such that

$$
\left\{\begin{array}{l}
\underline{I} \leq \liminf _{t \rightarrow+\infty} I(t) \leq I^{*} \leq \limsup _{t \rightarrow \infty} I(t) \leq \bar{I} \\
\frac{\gamma}{\mu+\delta}<1+\frac{\mu+\gamma}{\beta} \frac{\bar{h}(\underline{I}, \bar{I})-\underline{h}(\underline{I}, \bar{I})}{\bar{I}-\underline{I}}
\end{array}\right.
$$

and that

$$
\left(\begin{array}{l}
\underline{I} \leq \underline{I}^{*} \leq I^{*} \leq \bar{I}^{*} \leq \bar{I}, \\
\bar{I}^{*}+\frac{\mu+\gamma}{\beta} \bar{h}\left(\underline{I}^{*}, \bar{I}^{*}\right)=\frac{B}{\mu}-\frac{\gamma}{\mu+\delta} \underline{I}^{*}, \\
\text { and } \\
\underline{I}^{*}+\frac{\mu+\gamma}{\beta} \underline{h}\left(\underline{I}^{*}, \bar{I}^{*}\right)=\frac{B}{\mu}-\frac{\gamma}{\mu+\delta} \bar{I}^{*},
\end{array}\right) \quad \text { imply } \underline{I}^{*}=\bar{I}^{*}=I^{*} .
$$

Then, the positive equilibrium $E_{*}=\left(S^{*}, I^{*}, R^{*}\right)$ of system (1.2) is globally asymptotically stable in the interior of $\mathbb{R}_{+}^{3}$. In particular, if

$$
\left\{\begin{array}{l}
\underline{I} \leq \liminf _{t \rightarrow+\infty} I(t) \leq I^{*} \leq \limsup I(t) \leq \bar{I}, \\
\frac{\gamma}{\mu+\delta}<1+\frac{\mu+\gamma}{\beta} \frac{\bar{h}(\underline{\tilde{I}}, \tilde{\tilde{I}})-\underline{h}(\underline{\tilde{I}}, \overline{\tilde{I}})}{\overline{\tilde{I}}-\tilde{\tilde{I}}}, \\
\text { for any } \underline{\tilde{I}}<\overline{\tilde{I}} \text { such that } \underline{I} \leq \underline{\tilde{I}} \leq I^{*} \leq \overline{\tilde{I}} \leq \bar{I},
\end{array}\right.
$$

then, (1.11) and (1.12) is satisfied. 
Corollary 1.1 For $R_{0}>1$, if

$$
\gamma<\mu+\delta
$$

then the endemic equilibrium $E_{*}$ of system (1.2) is globally asymptotically stable in the interior of $\mathbb{R}_{+}^{3}$.

Note that a sufficient condition (1.14) for the endemic equilibrium to be globally asymptotically stable, is very simple and useful for a large class of SIRS models (1.2).

Let $R_{0}>1$. Then, by Theorem 1.2 , the positive equilibrium $E_{*}=\left(S^{*}, I^{*}, R^{*}\right)$ of system (1.2) exists. Assume that $0 \leq a_{0} \leq I^{*} \leq b_{0}$ and $G(I)$ is a unimodal function on $\left[a_{0}, b_{0}\right]$ and $\hat{I}$ is its maximal point of $G(I)$ on $\left[a_{0}, b_{0}\right]$, that is, $G(\hat{I})=\max _{a_{0} \leq I \leq b_{0}} G(I)$ and $G(I)$ is strictly monotone increasing on $\left[a_{0}, \hat{I}\right]$ and strictly monotone decreasing on $\left[\hat{I}, b_{0}\right]$. For simplicity, we suppose that $I^{*}<\hat{I}$ and $h(I)$ is a lower or upper convex function on $\left[a_{0}, b_{0}\right]$ and for any function $u(I)$ of $I$ on $\left[a_{0}, b_{0}\right]$, we use that if $I_{1}=I_{2}=I^{*}$, then $\frac{u\left(I_{1}\right)-u\left(I_{2}\right)}{I_{1}-I_{2}}$ means $u^{\prime}\left(I^{*}\right)$.

Corollary 1.2 Let $R_{0}>1$ and $G(I)$ be the above unimodal function on $\left[a_{0}, b_{0}\right]$ with $I^{*}<\hat{I}$.

If

$$
\frac{\beta}{\mu+\gamma} \hat{I}+\frac{\hat{I}}{G(\hat{I})}>R_{0}
$$

then $I^{*} \leq \lim \sup _{t \rightarrow \infty} I(t)<\hat{I}$.

Moreover, assume that there exist two constants $\underline{I}<\bar{I}$ such that

$$
\left\{\begin{array}{l}
a_{0} \leq \underline{I} \leq \liminf _{t \rightarrow+\infty} I(t) \leq I^{*} \leq \limsup _{t \rightarrow \infty} I(t) \leq \bar{I} \leq \hat{I}, \\
\frac{\gamma}{\mu+\delta}<1+\frac{\mu+\gamma}{\beta} \frac{h(\bar{I})-h(\underline{I})}{\bar{I}-\underline{I}}
\end{array}\right.
$$

and that

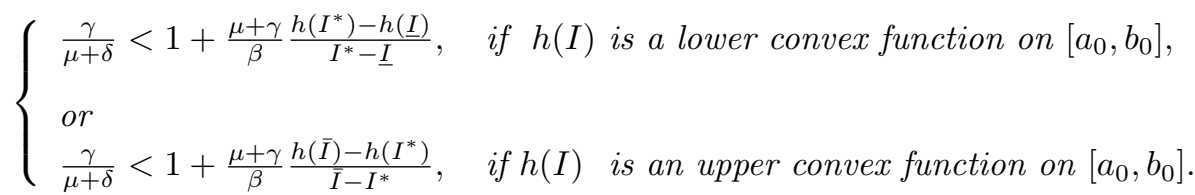

Then, the positive equilibrium $E_{*}=\left(S^{*}, I^{*}, R^{*}\right)$ of system (1.2) is globally asymptotically stable in the interior of $\mathbb{R}_{+}^{3}$.

For a particular case of $G(I)$ that

$$
G(I)=G_{p}(I) \equiv \frac{I}{1+\alpha I^{p}}, \quad \alpha>0, p>0,
$$

it holds that

$$
h(I)=I / G_{p}(I)=1+\alpha I^{p}, \quad \hat{I}= \begin{cases}\frac{1}{\sqrt[p]{(p-1) \alpha}}, & \text { if } p>1 \\ +\infty, & \text { if } p \leq 1 .\end{cases}
$$


We obtain the following corollary for the case (1.18) (cf. Xu and Ma [17, Theorem 3.1]).

Corollary 1.3 Let $G(I)$ be defined by (1.18) and $R_{0}>1$.

Then the positive equilibrium $E_{*}=\left(S^{*}, I^{*}, R^{*}\right)$ of system (1.2) exists.

If

$$
p>1 \quad \text { and } \quad \frac{\beta}{\mu+\gamma} \hat{I}+\frac{p}{p-1}>R_{0},
$$

then $\limsup _{t \rightarrow+\infty} I(t)<\hat{I}$.

Moreover, assume that there exist two constants $\underline{I}<\bar{I}$ such that

$$
0 \leq \underline{I} \leq \liminf _{t \rightarrow+\infty} I(t) \leq I^{*} \leq \limsup _{t \rightarrow \infty} I(t) \leq \bar{I}<\hat{I}
$$

and that

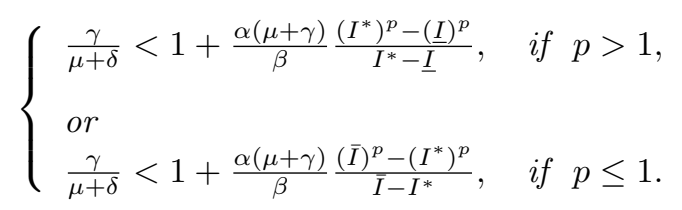

Then, the positive equilibrium $E_{*}=\left(S^{*}, I^{*}, R^{*}\right)$ of system (1.2) is globally asymptotically stable in the interior of $\mathbb{R}_{+}^{3}$.

Note that for $p=1, G(I)$ is monotone increasing on $\left[0, \limsup _{t \rightarrow \infty} I(t)\right]$ and the condition (1.22) becomes $\alpha(\mu+\gamma)(\mu+\delta)>\beta(\gamma-\mu-\delta)$ which greatly improves the condition $\alpha(\mu+\gamma)(\mu+\delta)>\beta(\gamma+\mu+\delta)$ in Xu and Ma [17, Theorem 3.1] (see also Section 4). Moreover, the result in Corollary 1.3 is a partial answer to the open problem proposed in Huo and Ma [5] and Yang and Xiao [18] such that for $1 \leq p \leq 2$, the positive equilibrium $E_{*}=\left(S^{*}, I^{*}, R^{*}\right)$ of system (1.2) with (1.18) is globally asymptotically stable in the interior of $\mathbb{R}_{+}^{3}$. In particular, all the examples for $R_{0}>1$ in Huo and Ma [5, Fig.3] satisfy (1.22), and the examples for $R_{0}>1$ in Yang and Xiao [18, Figures 3.6-3.8] that there exists a positive $\tau_{0}$ such that the endemic equilibrium $E_{*}$ of (1.2) can undergo Hopf bifurcation as $\tau>\tau_{0}$, and a periodic orbit appears in the small neighborhood of the endemic equilibrium $E_{*}$ under some conditions which do not satisfy (1.22).

The organization of this paper is as follows. In Section 2, we offer some basic results for system (1.2) and prove the local asymptotic stability of the disease-free equilibrium and prove Theorem 1.1. In Section 3, we first show the existence of the positive equilibrium of system (1.2) and prove the permanence of system (1.2). In Section 4, using monotone techniques similar to Xu and $\mathrm{Ma}$ [17], we prove Theorems 1.2, Corollaries 1.1-1.3. To illustrate our results, we offer numerical examples in Section 5. Finally, a short conclusion is offered in Section 6 . 


\section{Basic results}

We now state some basic results of system (1.2). Let $N(t)=S(t)+I(t)+$ $R(t)$.

Lemma 2.1 For system (1.2) with the initial condition (1.3),

$$
\lim _{t \rightarrow+\infty}(S(t)+I(t)+R(t))=\frac{B}{\mu} .
$$

Proof. It follows from system (1.2) that

$$
\begin{aligned}
\frac{d N(t)}{d t} & =B-\mu S(t)-\mu I(t)-\mu R(t) \\
& =B-\mu N(t) .
\end{aligned}
$$

Hence, we obtain that $\lim _{t \rightarrow+\infty} N(t)=B / \mu$. This completes the proof.

We now give the following lemmas concerning the local asymptotic stability of the disease-free equilibrium $E_{0}$ of system (1.2). This lemma is proved by using techniques in $\mathrm{Xu}$ and $\mathrm{Ma}$ [17].

Lemma 2.2 If $R_{0}<1$, then the disease-free equilibrium $E_{0}$ of system (1.2) is locally asymptotically stable. Furthermore, the disease-free equilibrium $E_{0}$ is unstable if $R_{0}>1$.

Proof. By $\lim _{I \rightarrow+0}\{I / G(I)\}=1$ in (1.4), we have that $\lim _{I(t-\tau) \rightarrow+0}\{I(t-\tau) / G(I(t-$ $\tau))\}=1$, and hence, the characteristic equation of system (1.2) at the diseasefree equilibrium $E_{0}$ is of the form (see Xu and Ma [17, Section 2])

$$
(\lambda+\mu)\left\{\lambda+(\mu+\gamma)\left(1-R_{0} \int_{0}^{h} k(\tau) \exp (-\lambda \tau) d \tau\right)\right\}(\lambda+\mu+\delta)=0 .
$$

Clearly, $\lambda=-\mu,-(\mu+\delta)$ are always roots of (2.2). All other roots of (2.2) are determined by the following equation.

$$
\lambda+(\mu+\gamma)\left(1-R_{0} \int_{0}^{h} k(\tau) \exp (-\lambda \tau) d \tau\right)=0 .
$$

We note that $\lambda=0$ is not a root of (2.3). When $\tau=0,(2.3)$ becomes as follows.

$$
\lambda+(\mu+\gamma)\left(1-R_{0}\right)=0 .
$$

If $R_{0}<1$, then one can see immediately that (2.4) has a negative real root. Therefore, the disease-free equilibrium $E_{0}$ of system (1.2) is locally asymptotically stable when $\tau=0$. Suppose that $\lambda=i \omega, \omega>0$ is a root of (2.3), 
separating real and imaginary parts, then we derive that

$$
\begin{aligned}
& (\mu+\gamma)\left(1-R_{0} \int_{0}^{h} k(\tau) \cos \omega \tau d \tau\right)=0 \\
& \omega+R_{0}(\mu+\gamma) \int_{0}^{h} k(\tau) \sin \omega \tau d \tau=0
\end{aligned}
$$

From the first equation of (2.5), we see that

$$
(\mu+\gamma)\left(1-R_{0} \int_{0}^{h} k(\tau) \cos \omega \tau d \tau\right) \geq(\mu+\gamma)\left(1-R_{0}\right)>0
$$

for all $\omega>0$, which is a contradiction. It follows that the real parts of all the eigenvalues of the characteristic equation (2.2) are negative for all $\tau \geq 0$. Therefore, if $R_{0}<1$, then the disease-free equilibrium $E_{0}$ of system (1.2) is locally asymptotically stable for all $\tau \geq 0$. Now, we put

$$
T(\lambda)=\lambda+(\mu+\gamma)\left(1-R_{0} \int_{0}^{h} k(\tau) \exp (-\lambda \tau) d \tau\right) .
$$

If $R_{0}>1$, then it is directly seen from (2.6) that for $\lambda \in \mathbb{R}$,

$$
T(0)=(\mu+\gamma)\left(1-R_{0}\right)<0, \lim _{\lambda \rightarrow+\infty} T(\lambda)=+\infty
$$

Therefore, (2.2) has at least one positive real root. Hence, if $R_{0}>1$, then the disease-free equilibrium $E_{0}$ is unstable. This completes the proof.

Proof of Theorem 1.1. From (2.1) in Lemma 2.1, for any $\epsilon>0$, there is a constant $T_{0} \geq 0$ such that

$$
S(t) \leq \frac{B}{\mu}+\epsilon \quad \text { for } t \geq T_{0} .
$$

Consider the following nonnegative function $W(t)$ defined by

$$
W(t)=I(t)+U(t), \quad t \geq T_{0}, \quad \text { for } \quad t \geq T_{0},
$$

where

$$
U(t)=\beta \int_{0}^{h} k(\tau) \int_{t-\tau}^{t} S(u+\tau) G(I(u)) d u d \tau, \quad t \geq T_{0} .
$$

Since for $t \geq T_{0}$,

$$
U^{\prime}(t)=\beta \int_{0}^{h} k(\tau)\{S(t+\tau) G(I(t))-S(t) G(I(t-\tau))\} d \tau
$$


we have that for $t \geq T_{0}$,

$$
\begin{aligned}
W^{\prime}(t)= & \left\{\beta S(t) \int_{0}^{h} k(\tau) G(I(t-\tau)) d \tau-(\mu+\gamma) I(t)\right\} \\
& +\beta \int_{0}^{h} k(\tau)\{S(t+\tau) G(I(t))-S(t) G(I(t-\tau))\} d \tau \\
= & \beta \int_{0}^{h} k(\tau) S(t+\tau) d \tau G(I(t))-(\mu+\gamma) I(t) \\
\leq & \beta \int_{0}^{h} k(\tau)\left(\frac{B}{\mu}+\epsilon\right) d \tau G(I(t))-(\mu+\gamma) I(t) \\
= & \left\{\frac{\beta B}{\mu}-(\mu+\gamma) \frac{I(t)}{G(I(t))}\right\} G(I(t))+\beta \epsilon G(I(t)) .
\end{aligned}
$$

Since $\epsilon>0$ is arbitrary, we obtain that if $R_{0}<1$, then $\frac{\beta B}{\mu}-(\mu+\gamma)<0$ and by (1.4), we have that $\frac{I}{G(I)}>1$ for $I>0$, and

$$
\begin{aligned}
W^{\prime}(t) & \leq\left\{\frac{\beta B}{\mu}-(\mu+\gamma) \frac{I(t)}{G(I(t))}\right\} G(I(t)) \\
& \leq\left\{\frac{\beta B}{\mu}-(\mu+\gamma)\right\} G(I(t)) \leq 0 .
\end{aligned}
$$

Then, the nonnegative function $W(t)$ is strictly monotone decreasing and there exists a nonnegative constant $\hat{W}$ such that $\lim _{t \rightarrow+\infty} W(t)=\hat{W}$. By the continuity of $G(I)$ and (2.8), we conclude that $\lim _{t \rightarrow+\infty} G(I(t))=\lim _{t \rightarrow+\infty} I(t)=0$ and $\hat{W}=0$. Thus, for $R_{0}<1$, we obtain from (1.2) that

$$
\lim _{t \rightarrow+\infty} S(t)=B / \mu, \quad \lim _{t \rightarrow+\infty} I(t)=0, \quad \text { and } \quad \lim _{t \rightarrow+\infty} R(t)=0 .
$$

It follows from Lemma 2.2 and Lyapunov-LaSalle asymptotic stability theorem that the disease-free equilibrium $E_{0}$ of system (1.2) is globally asymptotically stable if $R_{0}<1$.

\section{Permanence for $R_{0}>1$}

3.1. Existence and uniqueness of the endemic equilibrium $E_{*}$ of system (1.2) for $R_{0}>1$

By the condition (1.4), we also obtain the following basic lemma which ensures the existence and uniqueness of the endemic equilibrium $E_{*}$ of system (1.2) for $R_{0}>1$.

Lemma 3.1 If $R_{0}>1$, then system (1.2) has a unique endemic equilibrium $E_{*}$. 
Proof. Assume that $R_{0}>1$. From the second and the third equations of (1.6), it follows that

$$
S^{*}=\frac{(\mu+\gamma) I^{*}}{\beta G\left(I^{*}\right)}
$$

and

$$
R^{*}=\frac{\gamma I^{*}}{\mu+\delta} .
$$

After substituting (3.1) into the first equation of (1.6), we obtain that

$$
H\left(I^{*}\right)=0,
$$

where

$$
H(I) \equiv B-\frac{\mu(\mu+\gamma) I}{\beta G(I)}-(\mu+\gamma) I+\gamma \frac{\delta I}{\mu+\delta}=0 .
$$

By the condition of the monotonicity of $I / G(I)$ and $\lim _{I \rightarrow+0}(I / G(I))=1$ given in (1.4), one can see that $H(I)$ is a strictly monotone decreasing function of $I \in(0,+\infty)$, and

$$
\lim _{I \rightarrow+0} H(I)=B-\frac{\mu(\mu+\gamma)}{\beta}=B\left(1-\frac{1}{R_{0}}\right)>0,
$$

and for any $I>B /\left(\mu+\gamma\left\{1-\frac{\delta}{\mu+\delta}\right\}\right), H(I)<0$ holds. Hence, there exists a unique positive $I^{*}>0$ such that $H\left(I^{*}\right)=0$. By (3.1) and (3.2), there exists a unique endemic equilibrium $E_{*}=\left(S^{*}, I^{*}, R^{*}\right)$. Hence, the proof is complete.

\subsection{Permanence for $R_{0}>1$}

First, we prepare the following basic lemma.

Lemma 3.2 Assume that $I(s) \leq I^{*}$ for any $s$ such that $t-h \leq s<t$. If $I(t)<I(s)$ for any $s$ such that $t-h \leq s<t$ then $S(t)<S^{*}$. Inversely, if $S(t) \geq S^{*}$, then there exists an $s_{t} \in[t-h, t)$ such that $I(t) \geq I\left(s_{t}\right)$.

Proof. Assume that $I(t)<I(s) \leq I^{*}$ for any $s$ such that $t-h \leq s<t$. Then, $I^{\prime}(t)<0$ and by the second equation of (1.2) and the monotonicity of $\frac{I}{G(I)}$ for $I>0$, we have that

$$
\begin{aligned}
I^{\prime}(t) & =\beta S(t) \int_{0}^{h} k(\tau) G(I(t-\tau)) d \tau-(\mu+\gamma) I(t) \\
& \geq \int_{0}^{h} k(\tau)\{\beta S(t) G(I(t-\tau))-(\mu+\gamma) I(t-\tau)\} d \tau \\
& =\int_{0}^{h} k(\tau)\left\{\beta S(t)-(\mu+\gamma) \frac{I(t-\tau)}{G(I(t-\tau))}\right\} G(I(t-\tau)) d \tau \\
& \geq \int_{0}^{h} k(\tau)\left\{\beta S(t)-(\mu+\gamma) \frac{I^{*}}{G\left(I^{*}\right)}\right\} G(I(t-\tau)) d \tau \\
& =\beta\left(S(t)-S^{*}\right) \int_{0}^{h} k(\tau) G(I(t-\tau)) d \tau .
\end{aligned}
$$


Then, by $I^{\prime}(t)<0$ and $\int_{0}^{h} k(\tau) G(I(t-\tau)) d \tau>0$, we obtain that

$$
S(t)<S^{*}
$$

Inversely, assume that $I(s) \leq I^{*}$ for any $s$ such that $t-h \leq s<t$ and $S(t) \geq S^{*}$. Then, it is evident that there exists an $s_{t} \in[t-h, t)$ such that $I(t) \geq I\left(s_{t}\right)$.

Now, by applying Lemma 3.2, we offer a simplified proof for the permanence of system (1.2) than that of Wang [15] (see also Xu and Ma [17]).

Lemma 3.3 If $R_{0}>1$, then for any solution of system (1.2) with initial condition (1.3), it holds that

$$
\left\{\begin{array}{l}
\liminf \inf _{t \rightarrow+\infty} S(t) \geq v_{1}:=\frac{B}{\mu+\beta(B / \mu)}>0 \\
\liminf _{t \rightarrow+\infty} I(t) \geq v_{2}(q):=q G\left(I^{*}\right) \exp (-(\mu+\gamma) \rho(q))>0 \\
\liminf _{t \rightarrow+\infty} R(t) \geq v_{3}(q):=\frac{\gamma}{\mu+\delta} v_{2}(q)
\end{array}\right.
$$

where for any $0<q<1, \rho(q)>0$ is a constant such that

$$
S^{*}<S^{\triangle}:=\frac{B}{r}(1-\exp (-r \rho(q))), \quad \text { and } \quad r=\mu+\beta q G\left(I^{*}\right) .
$$

Proof. Let $(S(t), I(t), R(t))$ be any solution of system (1.2) with initial condition (1.3). By Lemma 2.1, it holds that

$$
\limsup _{t \rightarrow+\infty} I(t) \leq \frac{B}{\mu}
$$

For $\epsilon>0$ sufficiently small, there is a $T_{1}>0$ such that $I(t)<B / \mu+\epsilon$ for $t>T_{1}$. Then, by $G(I(t)) \leq I(t)$ and the first equation of $(1.2)$, we derive that

$$
S^{\prime}(t) \geq B-\{\mu+\beta(B / \mu+\epsilon)\} S(t),
$$

which implies that

$$
\liminf _{t \rightarrow+\infty} S(t) \geq \frac{B}{\mu+\beta(B / \mu+\epsilon)} .
$$

Since the above inequality holds for arbitrary $\epsilon>0$ sufficiently small, it follows that

$$
\liminf _{t \rightarrow+\infty} S(t) \geq \frac{B}{\mu+\beta(B / \mu)}=v_{1} .
$$

We now show that $\liminf _{t \rightarrow+\infty} I(t) \geq v_{2}(q)$ for any $0<q<1$. For any $0<q<1$, by (1.6), one can see that $S^{*}=\frac{B}{\mu+\beta G\left(I^{*}\right)}<\frac{B}{\mu+\beta q G\left(I^{*}\right)}=\frac{B}{r}$. Thus, there exist a positive constant $\rho(q)$ such that (3.4) holds.

We first prove the claim that it is not possible that for any solution $(S(t), I(t), R(t))$ of system (1.2), there exists a nonnegative constant $t_{0}$ such that $I(t) \leq q G\left(I^{*}\right)$ for all $t \geq t_{0}$. Suppose on the contrary that there exist a solution $(S(t), I(t), R(t))$ of system (1.2) and a nonnegative constant $t_{0}$ such 
that $I(t) \leq q G\left(I^{*}\right)$ for all $t \geq t_{0}$. Then, by $G(I) \leq I$ for $I>0$, we have that $I(t) \leq I^{*}$ for any $t \geq t_{0}$ and $G(I(t-\tau)) \leq I(t-\tau) \leq I^{*}$ for $t \geq t_{0}+h$ and $0 \leq \tau \leq h$, and from system (1.2), one can obtain that

$$
S^{\prime}(t) \geq B-\left(\mu+\beta q G\left(I^{*}\right)\right) S(t)=B-r S(t), \quad \text { for } t \geq t_{0}+h,
$$

which yields that

$$
\begin{aligned}
S(t) & \geq \exp \left(-r\left(t-t_{0}\right)\right)\left[S\left(t_{0}\right)+B \int_{t_{0}}^{t} \exp \left(r\left(\theta-t_{0}\right)\right) d \theta\right] \\
& \geq \frac{B}{r}\left\{1-\exp \left(-r\left(t-t_{0}\right)\right)\right\}, \quad \text { for any } t \geq t_{0}+h,
\end{aligned}
$$

Therefore, we have that

$$
S(t) \geq \frac{B}{r}\{1-\exp (-r \rho(q))\}=S^{\triangle}>S^{*}, \quad \text { for any } t \geq t_{0}+h+\rho(q) .
$$

Then, by the second part of Lemma 3.2, we obtain that $I^{\prime}(t) \geq 0$ and for any $t \geq t_{0}+h+\rho(q)$, there exists an $s_{t} \in[t-h, t)$ such that $I(t) \geq I\left(s_{t}\right)$. Therefore, for a positive constant $\hat{I}=\min _{t_{0}+\rho(q) \leq s \leq t_{0}+h+\rho(q)} I(s)$, we obtain that

$$
I(t) \geq \hat{I} \quad \text { for any } t \geq t_{0}+h+\rho(q) .
$$

Moreover, by $I(t) \leq q G\left(I^{*}\right) \leq q I^{*} \leq I^{*}$ for any $t \geq t_{0}+\rho$, we have that $G(I(t)) \geq \frac{G\left(I^{*}\right)}{I^{*}} I(t) \geq \frac{G\left(I^{*}\right)}{I^{*}} \hat{I}>0$ for any $t \geq t_{0}+\rho(q)$ and for the nonnegative function $W(t)$ defined by

$$
W(t)=I(t)+\beta \int_{0}^{h} k(\tau) \int_{t-\tau}^{t} S(u+\tau) G(I(u)) d u d \tau,
$$

we have that

$$
\begin{aligned}
W^{\prime}(t)= & \beta S(t) \int_{0}^{h} k(\tau) G(I(t-\tau)) d \tau-(\mu+\gamma) I(t) \\
& +\beta \int_{0}^{h} k(\tau)\{S(t+\tau) G(I(t))-S(t) G(I(t-\tau))\} \\
= & \beta \int_{0}^{h} k(\tau) S(t+\tau) G(I(t))-(\mu+\gamma) I(t) \\
= & \left\{\beta \int_{0}^{h} k(\tau) S(t+\tau)-(\mu+\gamma) \frac{I(t)}{G(I(t))}\right\} G(I(t)) \\
> & \left\{\beta S^{\triangle}-(\mu+\gamma) \frac{I^{*}}{G\left(I^{*}\right)}\right\} G(I(t)) \\
> & \beta\left\{S^{\triangle}-S^{*}\right\} \frac{G\left(I^{*}\right)}{I^{*}} \hat{I}>0, \text { for any } t \geq t_{0}+h+\rho(q),
\end{aligned}
$$

which implies that $\lim _{t \rightarrow+\infty} W(t)=+\infty$. However, by (3.7) and Lemma 2.1, there are a positive constant $t_{4} \geq t_{0}+h+\rho(q)$ and $\bar{W}$ such that $W(t) \leq \bar{W}$ for any $t \geq t_{4}$, which lead to contradiction. Hence, the claim is proved. 
By the claim, we are left to consider two possibilities. First, $I(t) \geq q G\left(I^{*}\right)$ for all $t$ sufficiently large. Second, $I(t)$ oscillates about $q G\left(I^{*}\right)$ for all $t$ sufficiently large.

We now show that $I(t) \geq v_{2}(q)$ for all $t$ sufficiently large. If the first condition that $I(t) \geq q G\left(I^{*}\right)$ holds for all sufficiently large, then we get the conclusion of the proof. For the second case that $I(t)$ oscillates about $q G\left(I^{*}\right)$ for all sufficiently large, let $t_{1}<t_{2}$ be sufficiently large such that

$$
I\left(t_{1}\right)=I\left(t_{2}\right)=q G\left(I^{*}\right), \quad \text { and } \quad I(t)<q G\left(I^{*}\right) \quad \text { for any } t_{1}<t<t_{2} .
$$

Then, by the second equation of system (1.2), we have that

$I(t) \geq-(\mu+\gamma) I(t)$, that is, $I(t) \geq I\left(t_{1}\right)\left\{1-\exp \left(-(\mu+\gamma)\left(t-t_{1}\right)\right)\right\}$, for any $t \geq t_{1}$,

from which, we have that for any $t \geq t_{1}$,

$$
I(t) \geq I\left(t_{1}\right)\left\{1-\exp \left(-(\mu+\gamma)\left(t-t_{1}\right)\right)\right\} \geq q G\left(I^{*}\right)\left\{1-\exp \left(-(\mu+\gamma)\left(t-t_{1}\right)\right)\right\} .
$$

Therefore, we obtain that

$$
I(t) \geq q G\left(I^{*}\right)\{-\exp (-(\mu+\gamma) \rho(q))\}=v_{2}(q), \quad \text { for any } t_{1} \leq t \leq t_{1}+\rho(q) .
$$

If $t_{2} \geq t_{1}+\rho(q)$, then by applying the similar discussion to (3.5) and (3.6) in place of $t_{0}$ by $t_{1}$, we obtain that $I(t) \geq v_{2}(q)$ for $t_{1}+\rho(q) \leq t \leq t_{2}$. Hence, we prove that $I(t) \geq v_{2}(q)$ for $t_{1} \leq t \leq t_{2}$. Since the interval $t_{1} \leq t \leq t_{2}$ is arbitrarily chosen, we conclude that $I(t) \geq v_{2}(q)$ for all sufficiently large for the second case. Since $q(0<q<1)$ is also arbitrarily chosen, Thus, we obtain that

$$
\liminf _{t \rightarrow+\infty} I(t) \geq v_{2}(q) .
$$

From the above discussion, one can see immediately that

$$
\liminf _{t \rightarrow+\infty} R(t) \geq v_{3}(q) .
$$

This completes the proof.

By Lemmas 2.1 and 3.3, we obtain the permanence of system (1.2) for $R_{0}>$ 1.

\section{Monotone iterative techniques to SIRS models}

In this section, for $R_{0}>1$, we improve the monotone iterative technique offered by $\mathrm{Xu}$ and $\mathrm{Ma}$ [17, Theorem 3.1] for system (1.2).

By Lemma 2.2, the existence of the endemic equilibrium $E_{*}$ of system (1.2) is guaranteed. 
Now, by (1.2) and Lemma 3.3, we may put

$$
\begin{cases}\liminf _{t \rightarrow+\infty} S(t)=\underline{\hat{S}} \geq v_{1}, & \liminf _{t \rightarrow+\infty} I(t)=\underline{\hat{I}} \geq v_{2}, \quad \liminf _{t \rightarrow+\infty} R(t)=\underline{\hat{R}} \geq v_{3}, \\ \limsup _{t \rightarrow+\infty} S(t)=\hat{\bar{S}} \leq \frac{B}{\mu}, & \limsup _{t \rightarrow+\infty} I(t)=\hat{\bar{I}} \leq \frac{B}{\mu}, \quad \limsup _{t \rightarrow+\infty} R(t)=\hat{\bar{R}} \leq \frac{B}{\mu} .\end{cases}
$$

By Lemma 2.1, hereafter, we may restrict our attention to the case that

$$
\lim _{t \rightarrow+\infty}(S(t)+I(t)+R(t))=\frac{B}{\mu}, \quad \text { for any } t \geq 0 .
$$

Then, we have the following lemma.

\section{Lemma 4.1}

$$
\frac{B}{\mu}-\underline{\hat{I}}-\hat{\bar{R}}>0, \quad \text { and } \quad \frac{B}{\mu}-\hat{\bar{I}}-\underline{\hat{R}}>0 .
$$

Proof. Suppose that $\frac{B}{\mu}-\hat{\bar{I}}-\underline{\hat{R}} \leq 0$. Then, by (4.1), there is a sequence $\left\{t_{n}\right\}_{n=1}^{\infty}$ such that $\lim _{n \rightarrow+\infty} I\left(t_{n}\right)=\hat{\bar{I}}$. Since $\liminf _{n \rightarrow+\infty} R\left(t_{n}\right) \geq \underline{\hat{R}}$, by (4.2), we have that

$$
0<\limsup _{n \rightarrow+\infty} S\left(t_{n}\right) \leq \frac{B}{\mu}-\liminf _{n \rightarrow+\infty} I\left(t_{n}\right)-\liminf _{n \rightarrow+\infty} R\left(t_{n}\right) \leq \frac{B}{\mu}-\hat{\bar{I}}-\underline{\hat{R}} \leq 0,
$$

which is a contradiction. Thus, we have $\frac{B}{\mu}-\hat{\bar{I}}-\underline{\hat{R}}>0$.

Similarly, we can prove that $\frac{B}{\mu}-\underline{\hat{I}}-\hat{\bar{R}}>0$.

\section{Lemma 4.2}

$$
\left\{\begin{array}{l}
0 \geq B-\mu \underline{\hat{S}}-\beta \underline{\hat{S}} \bar{G}(\underline{\hat{I}}, \hat{\bar{I}})+\delta\left(\frac{B}{\mu}-\underline{\hat{S}}-\hat{\bar{I}}\right), \\
0 \geq \beta\left(\frac{B}{\mu}-\underline{\hat{I}}-\hat{\bar{R}}\right) \underline{G}(\underline{\hat{I}}, \hat{\bar{I}})-(\mu+\gamma) \underline{\hat{I}}, \\
0 \geq \gamma \underline{\hat{I}}-(\mu+\delta) \underline{\hat{R}},
\end{array}\right.
$$

and

$$
\left\{\begin{array}{l}
0 \leq B-\mu \hat{\bar{S}}-\beta \hat{\bar{S}} \underline{G}(\underline{\hat{I}}, \hat{\bar{I}})+\delta\left(\frac{B}{\mu}-\hat{\bar{S}}-\hat{\underline{I}}\right) \\
0 \leq \beta\left(\frac{B}{\mu}-\hat{\bar{I}}-\underline{\hat{R}}\right) \bar{G}(\underline{\hat{I}}, \hat{\bar{I}})-(\mu+\gamma) \hat{\bar{I}} \\
0 \leq \gamma \hat{\bar{I}}-(\mu+\delta) \hat{\bar{R}}
\end{array}\right.
$$

Proof. Assume that $I(t)$ is eventually monotone decreasing for $t \geq 0$. Then, by Lemma (3.3), there exists $\lim _{t \rightarrow+\infty} I(t)=\hat{\bar{I}}=\hat{I}>0$. Then, by the third equation of (1.2), we obtain that there exists $\lim _{t \rightarrow+\infty} R(t)=\hat{\bar{R}}=\underline{\hat{R}}>0$. Then, by the first equation of (1.2), we obtain that there exists $\lim _{t \rightarrow+\infty} S(t)=\hat{\bar{S}}=\underline{\hat{S}}>0$. Since 
the positive equilibrium $E^{*}=\left(S^{*}, I^{*}, R^{*}\right)$ is unique, we have that $\hat{S}^{*}=\hat{\bar{S}}=\underline{\hat{S}}$, $\hat{I}^{*}=\hat{\bar{I}}=\hat{I}$ and $\hat{R}^{*}=\overline{\bar{R}}=\underline{\hat{R}}$. Thus, by (1.6), (4.5) holds.

Now, suppose that $I(t)$ is not eventually monotone decreasing for $t \geq 0$. Then, there exists a sequence $\left\{t_{n}\right\}_{n=1}^{\infty}$ such that $\lim _{n \rightarrow+\infty} I^{\prime}\left(t_{n}\right) \geq 0$ and $\lim _{n \rightarrow+\infty} I\left(t_{n}\right)=$ $\hat{\bar{I}}$. Since by (4.2),

$$
\limsup _{n \rightarrow+\infty} S\left(t_{n}\right) \leq \frac{B}{\mu}-\lim _{n \rightarrow+\infty} I\left(t_{n}\right)-\liminf _{n \rightarrow+\infty} R\left(t_{n}\right) \leq \frac{B}{\mu}-\hat{\bar{I}}-\underline{\hat{R}},
$$

we can immediately derive (4.5). Similarly, we can obtain (4.4). This completes the proof.

Then, we obtain that

$$
\left\{\begin{array}{l}
\underline{\hat{S}} \geq \frac{B\left(1+\frac{\delta}{\mu}\right)-\delta \hat{\bar{I}}}{(\mu+\delta)+\beta \bar{G}(\hat{\underline{I}}, \hat{I})} \\
\underline{\hat{I}}+\frac{\mu+\gamma}{\beta} \underline{h}(\underline{\hat{I}}, \hat{\bar{I}}) \geq \frac{B}{\mu}-\frac{\gamma}{\mu+\delta} \hat{\bar{I}} \\
\underline{\hat{R}} \geq \frac{\gamma}{\mu+\delta} \underline{\hat{I}}
\end{array}\right.
$$

and

$$
\left\{\begin{array}{l}
\hat{\bar{S}} \leq \frac{B\left(1+\frac{\delta}{\mu}\right)-\delta \hat{\underline{I}}}{(\mu+\delta)+\beta \underline{\underline{G}}(\underline{\underline{I}}, \hat{\bar{I}})} \\
\hat{\bar{I}}+\frac{\mu+\gamma}{\beta} \bar{h}(\underline{\hat{I}}, \hat{\bar{I}}) \leq \frac{B}{\mu}-\frac{\gamma}{\mu+\delta} \underline{\hat{I}} \\
\hat{\bar{R}} \leq \frac{\gamma}{\mu+\delta} \hat{\bar{I}}
\end{array}\right.
$$

We now consider the following four sequences $\bar{S}_{n}, \bar{I}_{n}, \underline{S}_{n}$ and $\underline{I}_{n},(n=1,2, \cdots)$ as follows (cf. Xu and Ma [17, (3.3)]).

$$
\left\{\begin{array}{l}
0 \leq \underline{I}_{0} \leq \liminf _{t \rightarrow+\infty} I(t) \\
\bar{K}\left(\underline{I}_{n-1}, \bar{I}_{n}\right)=\frac{B}{\mu}-\frac{\gamma}{\mu+\delta} \underline{I}_{n-1}, \\
\underline{K}\left(\underline{I}_{n}, \bar{I}_{n}\right)=\frac{B}{\mu}-\frac{\gamma}{\mu+\delta} \bar{I}_{n}, \quad n=1,2,3 \cdots
\end{array}\right.
$$

and

$$
\left\{\begin{array}{l}
\underline{S}_{n}=\frac{B(\mu+\delta) / \mu_{1}-\delta \bar{I}_{n}}{(\mu+\delta)+\beta \bar{G}\left(\underline{I}_{n}, \bar{I}_{n}\right)}, \quad \underline{R}_{n}=\frac{\gamma}{\mu+\delta} \underline{I}_{n}, \\
\bar{S}_{n}=\frac{B(\mu+\delta) / \mu_{1}-\delta \underline{I}_{n-1}}{(\mu+\delta)+\beta \underline{G}\left(\underline{I}_{n-1}, \bar{I}_{n}\right)}, \quad \bar{R}_{n}=\frac{\gamma}{\mu+\delta} \bar{I}_{n},
\end{array}\right.
$$

where the functions $\bar{K}(\underline{I}, \bar{I})$ and $\underline{K}(\underline{I}, \bar{I})$ are defined such that for any $0 \leq \underline{I} \leq \bar{I}$,

$$
\bar{K}(\underline{I}, \bar{I})=\bar{I}+\frac{\mu+\gamma}{\beta} \bar{h}(\underline{I}, \bar{I}), \quad \text { and } \quad \underline{K}(\underline{I}, \bar{I})=\underline{I}+\frac{\mu+\gamma}{\beta} \underline{h}(\underline{I}, \bar{I}) .
$$


Then, by Lemma $3.3,(4.7)$ and (4.8), we have that

$$
\underline{I}_{0} \leq \liminf _{t \rightarrow+\infty} I(t) \leq \limsup _{t \rightarrow+\infty} I(t) \leq \bar{I}_{1} .
$$

Lemma 4.3 For the sequences $\left\{\bar{I}_{n}\right\}_{n=1}^{\infty},\left\{\underline{I}_{n}\right\}_{n=1}^{\infty},\left\{\bar{S}_{n}\right\}_{n=1}^{\infty},\left\{\underline{S}_{n}\right\}_{n=1}^{\infty}$ defined by (4.8) and (4.9), assume $\underline{I}_{0}<\bar{I}_{1}$. Then,

$$
\underline{I}_{0}<\underline{I}_{1}<\bar{I}_{1}
$$

if and only if,

$$
\frac{\gamma}{\mu+\delta}<1+\frac{\mu+\gamma}{\beta} \frac{\bar{h}\left(\underline{I}_{0}, \bar{I}_{1}\right)-\underline{h}\left(\underline{I}_{1}, \bar{I}_{1}\right)}{\bar{I}_{1}-\underline{I}_{1}} .
$$

In this case, the three sequences $\left\{\underline{I}_{n}\right\}_{n=1}^{\infty},\left\{\underline{S}_{n}\right\}_{n=1}^{\infty}$ and $\left\{\underline{R}_{n}\right\}_{n=1}^{\infty}$ are strongly monotone increasing sequences and converge to $\underline{I}^{*}, \underline{S}^{*}$ and $\underline{R}^{*}$, respectively, and the three sequences $\left\{\bar{I}_{n}\right\}_{n=1}^{\infty},\left\{\bar{S}_{n}\right\}_{n=1}^{\infty}$ and $\left\{\bar{R}_{n}\right\}_{n=1}^{\infty}$ are strongly monotone decreasing sequences and converge to $\bar{I}^{*}, \bar{S}^{*}$ and $\bar{R}^{*}$, respectively, as $n$ tends to $+\infty$, and

$$
\left\{\begin{array}{l}
\lim _{n \rightarrow+\infty} \underline{I}_{n}=\underline{I}^{*} \leq \liminf _{t \rightarrow+\infty} I(t) \leq \limsup _{t \rightarrow+\infty} I(t) \leq \lim _{n \rightarrow+\infty} \bar{I}_{n}=\bar{I}^{*}, \\
\lim _{n \rightarrow+\infty} \underline{S}_{n}=\underline{S}^{*} \leq \liminf _{t \rightarrow+\infty} S(t) \leq \limsup _{t \rightarrow+\infty} S(t) \leq \lim _{n \rightarrow+\infty} \bar{S}_{n}=\bar{S}^{*}, \\
\lim _{n \rightarrow+\infty} \underline{R}_{n}=\underline{R}^{*} \leq \liminf _{t \rightarrow+\infty} R(t) \leq \limsup _{t \rightarrow+\infty} R(t) \leq \lim _{n \rightarrow+\infty} \bar{R}_{n}=\bar{R}^{*},
\end{array}\right.
$$

and

$$
\left\{\begin{array}{l}
\bar{I}^{*}+\frac{\gamma}{\mu+\delta} \underline{I}^{*}+\frac{\mu+\gamma}{\beta} \bar{h}\left(\underline{I}^{*}, \bar{I}^{*}\right)=\frac{B}{\mu} \\
\underline{I}^{*}+\frac{\gamma}{\mu+\delta} \bar{I}^{*}+\frac{\mu+\gamma}{\beta} \underline{h}\left(\underline{I}^{*}, \bar{I}^{*}\right)=\frac{B}{\mu}, \\
\text { and } \\
1+\frac{\mu+\gamma}{\beta} \frac{\bar{h}\left(\underline{I}^{*}, \bar{I}^{*}\right)-\underline{h}\left(I^{*}, \bar{I}^{*}\right)}{\bar{I}^{*}-\underline{I}^{*}}=\frac{\gamma}{\mu+\delta}, \text { if } \underline{I}^{*}<\bar{I}^{*}, \\
I^{*} \leq \hat{I}, \text { if } \underline{I}^{*}=\bar{I}^{*}=I^{*} .
\end{array}\right.
$$

Moreover, if (1.11) and (1.12) hold, then

$$
\underline{I}^{*}=\bar{I}^{*}=I^{*} \leq \hat{I}, \quad \underline{S}^{*}=\bar{S}^{*}=S^{*} \quad \text { and } \quad \underline{R}^{*}=\bar{R}^{*}=R^{*} .
$$

In particular, if

$$
\frac{\gamma}{\mu+\delta} \leq 1
$$

then (4.16) holds.

Proof. By (4.8) and (4.10),

$$
\left\{\begin{array}{l}
\bar{I}_{n}+\frac{\mu+\gamma}{\beta} \bar{h}\left(\underline{I}_{n-1}, \bar{I}_{n}\right)=\frac{B}{\mu}-\frac{\gamma}{\mu+\delta} \underline{I}_{n-1}, \\
\underline{I}_{n}+\frac{\mu+\gamma}{\beta} \underline{h}\left(\underline{I}_{n}, \bar{I}_{n}\right)=\frac{B}{\mu}-\frac{\gamma}{\mu+\delta} \bar{I}_{n}, \quad n=1,2,3, \cdots,
\end{array}\right.
$$


from which we have that for $\underline{I}_{n}<\bar{I}_{n}$ and $n=1,2,3, \cdots$,

$$
\left(1+\frac{\mu+\gamma}{\beta} \frac{\bar{h}\left(\underline{I}_{n-1}, \bar{I}_{n}\right)-\underline{h}\left(\underline{I}_{n}, \bar{I}_{n}\right)}{\bar{I}_{n}-\underline{I}_{n}}\right)\left(\bar{I}_{n}-\underline{I}_{n}\right)=\frac{\gamma}{\mu+\delta}\left(\bar{I}_{n}-\underline{I}_{n-1}\right) .
$$

Hence, we obtain that for $\underline{I}_{n}<\bar{I}_{n}$,

$$
\bar{I}_{n}-\underline{I}_{n}=\frac{\frac{\gamma}{\mu+\delta}}{1+\frac{\mu+\gamma}{\beta} \frac{\bar{h}\left(\underline{I}_{n-1} \bar{I}_{n}\right)-\underline{h}\left(\underline{I}_{n}, \bar{I}_{n}\right)}{\bar{I}_{n}-\underline{I}_{n}}}\left(\bar{I}_{n}-\underline{I}_{n-1}\right), \quad n=1,2,3, \cdots,
$$

from which one can see that (4.12) holds, if and only if, (4.13) holds. Then, by the monotonicity and inductions in (4.18), we can prove that $\underline{I}_{n-1}<\underline{I}_{n}<\bar{I}_{n}<$ $\bar{I}_{n-1}, n=2,3, \cdots,(4.14)$ and (4.15) hold. Moreover, suppose that (1.11) and (1.12) hold. Then, $I^{*} \leq \hat{I}$ and by (4.19), we obtain (4.16). Hence, by (4.6) and (4.7), we obtain the conclusion of this lemma.

Proof of Theorem 1.2. By Lemma 4.3, we obtain the conclusion of Theorem 1.2 .

Now, we give a property of a lower or upper convex function of $I$ on $\left[a_{0}, b_{0}\right]$.

Lemma 4.4 If $h(I)$ is a lower or upper convex function on $\left[a_{0}, b_{0}\right]$, then for any $a_{0} \leq \underline{I} \leq I^{*} \leq \bar{I} \leq b_{0}$,

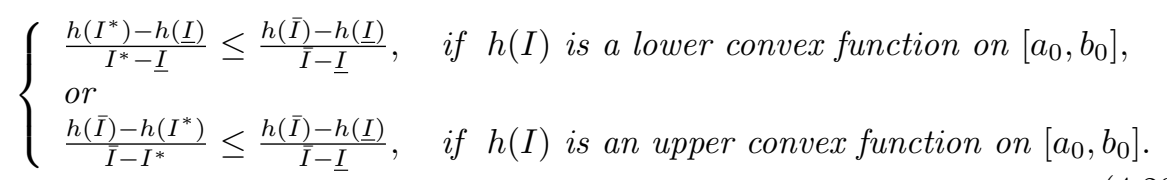

Proof. The proof of this lemma is evident from the definition of a lower and upper convex function of $I$ on $\left[a_{0}, b_{0}\right]$.

Proof of Corollaries 1.1-1.3. Since by assumption that $I^{*}<\hat{I}$ and the first equation of (4.15) in Lemma 4.3, we have that

$$
\frac{\beta}{\mu+\gamma} \bar{I}^{*}+\bar{h}\left(\underline{I}^{*}, \bar{I}^{*}\right)=R_{0}-\frac{\beta}{\mu+\gamma} \frac{\gamma}{\mu+\delta} \underline{I}^{*}<R_{0} .
$$

Therefore, from (1.15), one can easily see that $\bar{I}^{*}<\hat{I}$. Then, by using the results in Theorem 1.2 with Lemmas 4.3 and 4.4, we immediately obtain the conclusion of Corollaries 1.1-1.3.

For the cases $p=1$ and $p>1$, by Corollary 1.3, we easily obtain the following result.

Corollary 4.1 Let $p \geq 1$ and $R_{0}>1$. If

$$
\{\alpha(\mu+\gamma)+\beta\}(\mu+\delta)-\beta \gamma>0, \quad \text { for } p=1,
$$


or (1.20)-(1.22) hold for $p>1$, then the positive equilibrium $E_{*}=\left(S^{*}, I^{*}, R^{*}\right)$ of system (1.2) with (1.18) is globally asymptotically stable in the interior of $\mathbb{R}_{+}^{3}$. In particular, for $p=2$, if

$$
\frac{\beta}{(\mu+\gamma) \sqrt{\alpha}}+2>R_{0} \quad \text { and } \quad \frac{\mu+\delta}{\gamma}\left(1+\frac{\mu+\gamma}{\beta} I^{*}\right)>1
$$

then, conditions (1.20)-(1.22) are satisfied.

Note that the sufficient condition in Xu and Ma [17, Theorem 3.1] for $p=1$ becomes $\alpha(\mu+\gamma)(\mu+\delta)>\beta(\gamma+\mu+\delta)$, but the condition (4.22) is $\alpha(\mu+\gamma)(\mu+$ $\delta)>\beta(\gamma-\mu-\delta)$. Moreover, for the case $p=2$ and $R_{0}>1$, we solve the open question to an example in Huo and Ma [5, Example], because one can see that this example satisfies the condition (4.22) in Corollary 4.1 see Muroya et al. [12, Theorem 1.1].

\section{Numerical examples}

In this section, we restrict our attention only to the following case in system (1.2):

$$
\left\{\begin{array}{l}
G(I)=\frac{I}{1+\alpha I}, \\
B=2, \quad \alpha=1, \quad \gamma=0.1, \quad \mu=0.3, \quad \delta=0.2, \\
h=10, \quad \text { and } \quad k(\tau)=0.1,
\end{array}\right.
$$

and $\beta>0$ will be determined later.

By Theorem 1.1, we see that the disease-free equilibrium $E_{0}$ of system (1.2) is globally asymptotically stable if $R_{0}<1$ which is equivalent to $\beta<0.06$. Figure 1 for the case (5.1) with $\beta=0.05<0.06$, indicates that $R_{0}=0.833 \cdots<1$ and the disease-free equilibrium $E_{0}=(6.666 \cdots, 0,0)$ of system $(1.2)$ is globally asymptotically stable.

By Corollary 4.1, we see that the endemic equilibrium $E^{*}$ of system (1.2) is globally asymptotically stable if $R_{0}>1$ and it holds (4.21) in Corollary 4.1, which is equivalent to $0.06<\beta<0.88$. On the other hand, the sufficient condition for the global asymptotic stability of the endemic equilibrium of system (1.2) in $\mathrm{Xu}$ and $\mathrm{Ma}$ [17] becomes $0.06<\beta<0.333 \cdots$. Thus, the condition (4.21) in Corollary 4.1 greatly improves the condition in $\mathrm{Xu}$ and $\mathrm{Ma}$ [17]. Figure 2 gives a graph trajectory of $\underline{I}_{n}$ and $\bar{I}_{n}$ for the case (5.1) with $\beta=0.5$ and the starting value $\underline{I}_{0}=0$ in (4.8), indicates that $R_{0}=8.333 \cdots>1$ and both $\bar{I}_{n}$ and $\underline{I}_{n}(n \geq 1)$ defined by $(4.8)$ converge to $I^{*}=2.933 \cdots$, and Figure 3 indicates that the endemic equilibrium $E^{*}=(3.146 \cdots, 2.933 \cdots, 0.586 \cdots)$ of system (1.2) is globally asymptotically stable.

\section{Conclusion}

In this paper, for SIRS epidemic models with a class of nonlinear incidence rates and distributed delays of the forms $\beta S(t) \int_{0}^{h} k(\tau) G(I(t-\tau)) d \tau$, we establish 


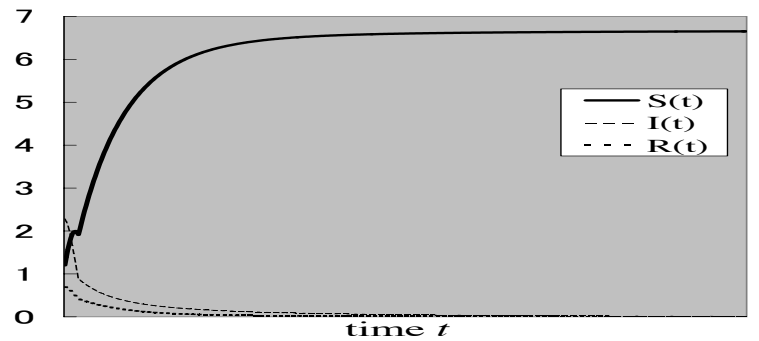

Figure 1: A graph trajectory of $S(t), I(t)$ and $R(t)$ of system (1.2) for the case (5.1) with $\beta=0.05$. We have $R_{0}=0.833 \cdots<1$ and $E_{0}=(6.666 \cdots, 0,0)$.

the global asymptotic stability of the disease-free equilibrium $E_{0}$ for $R_{0}<1$, and applying new monotone iterative techniques, we obtain sufficient conditions for the global asymptotic stability of the endemic equilibrium of systems (1.2) for $R_{0}>1$, respectively. In particular, by applying Lemma 3.2, we offer a simplified proof for the permanence of system (1.2) than that of Wang [15] (see also $\mathrm{Xu}$ and $\mathrm{Ma}[17])$. We note that a sufficient condition (1.14) obtained by a simple conditions of contractive convergence for suitable monotone iterations (see (4.8)), for the endemic equilibrium to be globally asymptotically stable, is very useful for a large class of SIRS models (1.2).

We also note that the conditions (1.4) and (1.13) play important roles to obtain the global asymptotic stability of the endemic equilibrium $E_{*}$ of systems (1.2) for $R_{0}>1$, respectively. Moreover, by the sake of Lemma 4.2, our monotone iterative techniques become much improved one than that in $\mathrm{Xu}$ and $\mathrm{Ma}$ [17] which was applied to the saturated incidence rate $G(I)=\frac{I}{1+\alpha I}$.

As a result, we have solved the conjecture to the example in Huo and Ma [5] that the endemic equilibrium of system (1.2) is globally asymptotically stable if $R_{0}>1$, and also offer partial answers to the open problem in Huo and Ma [5] and Yang and Xiao [18].

These techniques are also applicable to various kinds of epidemic models with delays. These will be our future works.

[1] R. M. Anderson and R. M. May, Population biology of infectious diseases: Part I, Nature 280 (1979) 361-367.

[2] E. Beretta, T. Hara, W. Ma and Y. Takeuchi, Global asymptotic stability 


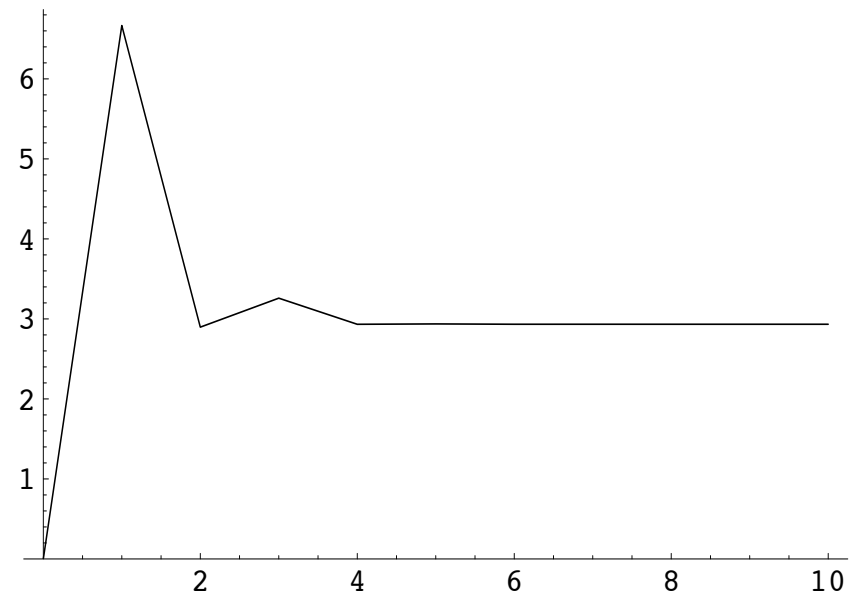

Figure 2: A graph trajectory of $\underline{I}_{n}$ and $\bar{I}_{n}\left(0=\underline{I}_{0} \rightarrow \bar{I}_{1} \rightarrow \underline{I}_{1} \rightarrow \cdots\right)$ defined by (4.8) for the case (5.1) with $\beta=0.5$.

of an SIR epidemic model with distributed time delay, Nonlinear Anal. 47 (2001) 4107-4115.

[3] V. Capasso and G. Serio, A generalization of the Kermack-Mckendrick deterministic epidemic model, Math. Biosci. 42 (1978) 41-61.

[4] K. L. Cooke, Stability analysis for a vector disease model, Rocky Mountain J. Math. 9 (1979) 31-42.

[5] Hai-Feng Huo and Zhan-Ping Ma, Dynamics of a delayed epidemic model with non-monotonic incidence rate, Commun Nonlinear Sci Numer Simulat 15 (2010), 459-468.

[6] Y. Jin, W. Wang and S. Xiao, An SIRS model with a nonlinear incidence rate, Chaos, Solitons and Fractals. 34 (2007) 1482-1497.

[7] A. Korobeinikov, Global Properties of Infectious Disease Models with Nonlinear Incidence, Bull. Math. Biol. 69 (2007) 1871-1886.

[8] W. M. Liu, S. A. Levin and Y. Iwasa, Influence of nonlinear incidence rates upon the behavior of SIRS epidemiological models. J. Math. Biol. 23 (1986), 187-204.

[9] W. Ma, M. Song and Y. Takeuchi, Global stability of an SIR epidemic model with time delay, Appl. Math. Lett. 17 (2004) 1141-1145.

[10] C. C. McCluskey, Complete global stability for an SIR epidemic model with delay-Distributed or discrete, Nonl. Anal. RWA. 11 (2010) 55-59.

[11] J. Mena-Lorca and H. W. Hethcote, Dynamic models of infectious diseases as regulators of population size, J. Math. Biol. 30 (1992) 693-716. 


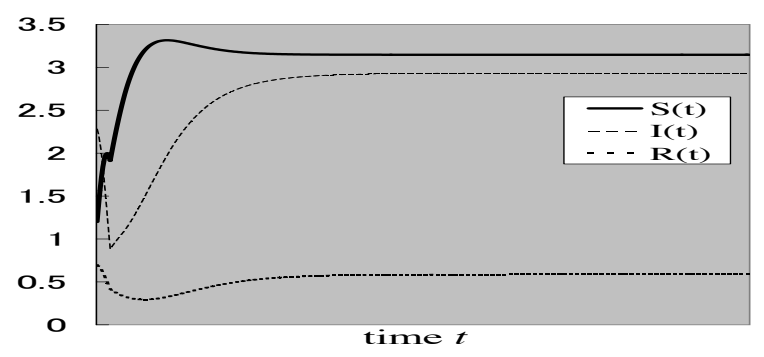

Figure 3: A graph trajectory of $S(t), I(t)$ and $R(t)$ of system (1.2) for the case (5.1) with $\beta=0.5$. We have $R_{0}=8.333 \cdots>1$ and $E^{*}=(3.146 \cdots, 2.933 \cdots, 0.586 \cdots)$.

[12] Y. Muroya, Y. Enatsu and Y. Nakata, Global stability of a delayed SIRS epidemic model with a non-monotonic incidence rate, submitted

[13] G. Pang and L. Chen, A delayed SIRS epidemic model with pulse vaccination, Chaos, Solitons and Fractals, 34 (2007) 1629-1635.

[14] Y. Takeuchi, W. Ma and E. Beretta, Global asymptotic properties of a delay SIR epidemic model with finite incubation times, Nonlinear Anal. 42 (2000) 931-947.

[15] W. Wang, Global behavior of an SEIRS epidemic model with time delays, Appl. Math. Lett. 15 (2002) 423-428.

[16] D. Xiao and S. Ruan, Global analysis of an epidemic model with nonmonotone incidence rate, Math. Biosci. 208 (2007) 419-429.

[17] R. Xu and Z. Ma, Stability of a delayed SIRS epidemic model with a nonlinear incidence rate, Chaos, Solitons and Fractals. 41 (2009) 23192325 .

[18] Y. Yang and D. Xiao, Influence of latent period and nonlinear incidence rate on the dynamics of SIRS epidemiological models, Discrete and Continuous Dynamical Systems Series B 13 (2010), 195-211. 